\title{
Entre los marcos jurídicos y las cartografías indígenas
}

Una revisión de conceptos en torno a la soberanía nacional en Venezuela

Cadres juridiques et cartographies autochtones: une révision des concepts

autour de la souveraineté nationale au Venezuela

Between legal frameworks and indigenous cartographies: A review of concepts

regarding national sovereignty in Venezuela

\section{Hortensia Caballero Arias}

\section{OpenEdition \\ Journals}

Edición electrónica

URL: http://journals.openedition.org/ethnoecologie/2633

DOI: 10.4000/ethnoecologie.2633

ISSN: 2267-2419

Editor

Laboratoire Eco-anthropologie et Ethnobiologie

\section{Referencia electrónica}

Hortensia Caballero Arias, «Entre los marcos jurídicos y las cartografías indígenas », Revue

d'ethnoécologie [En ligne], 9 | 2016, mis en ligne le 01 juillet 2016, consulté le 30 avril 2019. URL : http:// journals.openedition.org/ethnoecologie/2633; DOI : 10.4000/ethnoecologie.2633

Este documento fue generado automáticamente el 30 abril 2019.

Revue d'ethnoécologie est mis à disposition selon les termes de la licence Creative Commons Attribution - Pas d'Utilisation Commerciale - Pas de Modification 4.0 International. 


\title{
Entre los marcos jurídicos y las cartografías indígenas
}

\author{
Una revisión de conceptos en torno a la soberanía nacional en Venezuela \\ Cadres juridiques et cartographies autochtones : une révision des concepts \\ autour de la souveraineté nationale au Venezuela \\ Between legal frameworks and indigenous cartographies: A review of concepts \\ regarding national sovereignty in Venezuela
}

\author{
Hortensia Caballero Arias
}

\section{Introducción}

1 Las cartografías sociales indígenas, los mapeos participativos o las etnocartografías se han convertido en una poderosa herramienta visual para que las poblaciones locales reconstruyan y representen sus espacios y territorios desde sus propias visiones del mundo. La literatura es copiosa y ha tratado de dar cuenta de los aspectos cognitivos y fenomenológicos percibidos por los indígenas sobre los territorios que ocupan en cuanto a sus actividades de subsistencia, cosmogonía, conocimiento de los recursos fluviales y faunísticos, sitios sagrados, biodiversidad, prácticas lúdicas, entre otros ${ }^{1}$. Sin embargo, las cartografías indígenas o mapas mentales o culturales también se han constituido en instrumentos políticos para la defensa de sus territorios e identidades ante la expansión de agentes externos. Este trabajo plantea explorar esa dimensión política de las demandas territoriales indígenas con respecto al Estado-nación venezolano. En particular, propone discutir los procesos de demarcación de tierras y las cartografías indígenas en relación con los marcos jurídicos nacionales y contrastarlos con la noción de soberanía nacional en Venezuela.

Para ello, presentaremos en primer lugar un contexto general de las luchas y estrategias heurísticas de disputa territorial, y la significación holística que tienen ciertas fechas conmemorativas para los indígenas en América Latina en relación con la Pachamama. 
Luego discutiremos los marcos jurídicos vigentes que reconocen los derechos de los pueblos indígenas para analizar cómo los conceptos que expone la Ley Orgánica de Pueblos y Comunidades Indígenas (LOPCI 2005) en Venezuela en torno a "pueblos y comunidades indígenas", por una parte, y «tierras y hábitats indígenas » por la otra no vulneran la noción de soberanía nacional ni contradicen los principios de la geopolítica nacional, como se ha pretendido establecer.

3 Asimismo, planteamos que los mapas mentales o culturales indígenas que forman parte de los requerimientos para hacer la solicitud formal de las tierras ante las instancias gubernamentales reflejan una visión espacial intercultural que toma en cuenta tanto las visiones territoriales indígenas como el ordenamiento político-administrativo de la nación venezolana. Los mapas mentales indígenas son objetos de producción sociohistórica que no contrarían la idea de la soberanía nacional y por el contrario, constituyen ejemplos patrimoniales y estéticos de conocimiento espacial y territorial que pueden dar cuenta de un sentido más político de la idea de nación.

\section{Día de la Resistencia Indígena: Estrategias en torno a las disputas territoriales}

4 En las últimas dos décadas se ha hecho habitual que las organizaciones indígenas en diferentes países de América Latina conmemoren los 12 de octubre, no como día festivo sino como el Día de la Resistencia en el marco de la llegada de los europeos a este continente hace más de 500 años. En estos actos públicos, los indígenas rememoran y condenan las acciones genocidas y etnocidas que se llevaron a cabo durante la conquista y la colonización europea, y cuestionan de manera reiterada las viejas interpretaciones eufemísticas del llamado "Día de la Raza », «Día del descubrimiento de América » o el «Encuentro de Dos Mundos». En ese espacio de remembranza histórica y política, los indígenas de las Américas aprovechan también para protestar en contra de las formas de dominación y discriminación a las que están actualmente expuestos como consecuencia del impacto indiscriminado de la extracción minera, petrolera y forestal, así como la indefensión en la que muchos pueblos indígenas se encuentran ante la continua expansión de colonos, mineros y ganaderos sobre sus tierras y áreas de ocupación².

5 Es así como en la actualidad esta fecha histórica ha adquirido diversos nombres y significados que obedecen a la apertura multicultural de los Estados, la cual se manifiesta en el reconocimiento de la diversidad cultural dentro de las naciones y la visibilización de los movimientos etno-políticos indígenas. En Venezuela, por ejemplo, lleva ahora el nombre de Día de la Resistencia Indígena ${ }^{3}$, en Argentina es el Día del Respeto a la Diversidad Cultural, en Perú es el Día de los Pueblos Originarios y del Diálogo Intercultural, y en Ecuador es el Día de la Interculturalidad y la Plurinacionalidad, entre otros. Estas diferencias nominales que cada país le ha asignado a esta celebración institucionalizada representan una apertura no sólo para repensar y cuestionar los procesos históricos de conquista y colonización, sino también para exigir sus derechos como pueblos indígenas, quienes a su vez juegan un papel relevante en las actuales dinámicas socio-políticas latinoamericanas.

6 La conmemoración de los 12 de octubre se ha constituido en un momento y escenario político para realizar una serie de demostraciones, cuestionamientos y demandas territoriales por parte de organizaciones y representantes indígenas. Por ejemplo, en 
Chile los indígenas mapuches protestan en contra de la militarización de sus territorios; los pueblos indígenas del Cauca en Colombia a través del Consejo Regional Indígena del Cauca (CRIC) marchan en defensa de la Madre Tierra; y la Coordinadora Andina de Organizaciones Indígenas (CAOI) con otras organizaciones indígenas de América Latina se han pronunciado en repetidas ocasiones en defensa y cuidado de las tierras indígenas, conocida como la Pachamama. La Pachamama (también llamada Mama Pacha), que se traduce del quechua como "Madre Tierra", es una deidad incaica de los pueblos indígenas de los Andes Centrales que representa la tierra y que se ha constituido en un vocablo alegórico en la defensa de las tierras indígenas en la mayor parte del continente.

7 En el caso de Venezuela, esta fecha también se ha considerado emblemática entre los pueblos indígenas quienes aprovechan la oportunidad para reunirse y elevar sus demandas en relación con las solicitudes de demarcación de sus tierras desde la promulgación de la Ley de Demarcación y Garantía del Hábitat y Tierras de los Pueblos Indígenas (LDTPI 2001). Cada año, indígenas de diferentes etnias se congregan en Ciudad Bolívar, estado Bolívar; en Puerto Ayacucho, estado Amazonas y en diferentes poblaciones del estado Zulia para realizar movilizaciones exhortando a las instituciones públicas y a las comisiones oficiales de trabajo a acelerar los procesos de demarcación de las tierras y hábitats indígenas en Venezuela. La asignación de tierras a los pueblos indígenas es una tarea pendiente en la agenda gubernamental de la revolución bolivariana a pesar de que se han otorgado algunos títulos de tierras a "comunidades indígenas »4. Sin embrago, conocemos, por ejemplo que las supuestas concesiones de tierras por parte del Estado ${ }^{5}$ a los indígenas de la Sierra de Perijá, en el Zulia, han sido el resultado de largas luchas, conflictos, atropellos e incluso asesinatos de dirigentes indígenas ${ }^{6}$ que los mismos yukpa han denunciado incluso ante organismos internacionales al exigir el reconocimiento de los derechos sobre sus tierras ancestrales (Portillo 2011, Romero 2012).

Esto significa que la preocupación de los pueblos indígenas en Venezuela en reclamar los derechos sobre sus tierras sigue siendo una prioridad entre sus demandas socio-culturales ante el Estado bolivariano. De tal manera, nos parece comprensible y predecible que ante las dificultades crecientes en los procesos de demarcación territorial que han experimentado los pueblos indígenas en Venezuela en los últimos quince años, la Universidad Indígena de Venezuela del Caño Tauca, en el estado Bolívar hiciera una declaración fehaciente el Día de la Resistencia Indígena en el 2011, al afirmar que « hay que ser firme a la hora de defender a la Pachamama » (UIV 2011). Este vocablo quechua que ha traspasado las fronteras andinas, se ha convertido en la actualidad en un símbolo de lucha y representación colectiva de las solicitudes de tierras entre los pueblos indígenas de América Latina.

9 Tomando prestada la categoría literaria de « cronotopo » de Bajtin (1989) que refiere a ese vínculo esencial de relaciones temporales y espaciales asimiladas artísticamente, podríamos decir que la asociación entre un tiempo conmemorativo como el Día de la Resistencia Indígena y un referente espacial panamericano como la Pachamama se han constituido en un cronotopo político de lucha Amerindia. Las acciones en defesa y protección de las tierras, de la Pachamama en términos genéricos, se han revelado a través de estrategias heurísticas de disputa que reclaman la protección de la naturaleza, el reconocimiento de los derechos indígenas sobre sus territorios, y el respecto a los modos indígenas de percibir y representar sus territorios espacialmente.

10 Cuando señalamos que la evocación, reclamo y resguardo de la Pachamama desde una perspectiva Amerindia constituye una estrategia heurística de disputa por parte de los 
pueblos indígenas en América Latina, nos estamos refiriendo a que las luchas y reclamos de tierras han sido el resultado de movilizaciones sociales indígenas creativas y diversas que buscan evaluar y colectivizar sus problemas y demandas sociales. Estas acciones proponen resolver conflictos territoriales, evitar los desplazamientos y exigir el reconocimiento a sus derechos territoriales ante los Estados-naciones a partir de metodologías interculturales que tomen en cuenta las particularidades étnicas, la especificidad ecológica y los vínculos que ellos han mantenido ancestralmente con sus territorios. Esas estrategias heurísticas de contienda también se refieren a la participación directa de los indígenas en el desarrollo de propuestas de gobernanzas territoriales y al diseño de cartografías sociales indígenas que son herramientas visuales y políticas para posicionarse territorialmente dentro de los Estados-naciones ${ }^{7}$. Esas capacidades y mecanismos que desarrollan los indígenas para reivindicar sus derechos originarios como pueblos, ha permitido desentrañar viejos prejuicios etnocentristas sobre la soberanía nacional, así como evidenciar las nuevas formas de exclusión y colonización como es el caso de la expansión del neoextractivismo en muchos territorios indígenas de América Latina.

11 Si bien algunos países, en cierta medida, han superado las limitaciones impuestas por los aparatos burocráticos del Estado en cuanto al reconocimiento de las tierras indígenas, hay otros casos en los que todavía los pueblos indígenas no han sido del todo beneficiados por las políticas de inclusión del multiculturalismo, a pesar de sus novedosas constituciones pluriétnicas. El caso de Venezuela en relación con a la demarcación y titulación de tierras indígenas resulta ser paradójico y contrastante en cuanto a su limitado alcance. Mientras el Estado bolivariano ha reconocido los derechos originarios de los pueblos indígenas en su Constitución Nacional de 1999 y ha declarado una serie de leyes y normativas que consideran y respaldan las tradiciones y culturas indígenas, hasta el presente solo una pequeña porción de " comunidades indígenas » han sido favorecidas con la titulación de las tierras. Existe, por lo tanto, una brecha entre lo jurídico y lo fáctico, o lo que algunos autores han identificado como una resistencia gubernamental ante la titulación de grandes extensiones de tierra para « pueblos indígenas » (Caballero Arias 2007, Mosonyi 2009, Angosto 2010).

En estos actuales escenarios de reconocimiento de los derechos indígenas en Venezuela vale la pena preguntarse entonces ¿de qué manera los pueblos indígenas ejercen la libre determinación y el derecho a la autonomía en cuanto al uso y manejo de sus tierras, tal como lo consagra la Declaración de las Naciones Unidas sobre los Derechos de los Pueblos Indígenas (2007)?, ¿cómo los indígenas articulan sus visiones sobre las tierras y hábitats que tradicionalmente han ocupado con la idea de nación venezolana?, ¿es posible que las cartografías indígenas puedan dar cuenta de la dimensión geopolítica del territorio y reflejar criterios sobre la soberanía nacional?

Estas preguntas nos llevan fundamentalmente a interrogar la dimensión más política de las demarcaciones de tierras indígenas. Así, en los próximos apartados examinaremos los marcos jurídicos vigentes sobre los derechos colectivos indígenas en Venezuela. Al mismo tiempo evaluaremos las razones geopolíticas por las que el proceso de demarcación de tierras indígenas en Venezuela ha sido tan accidentado y desestimado por algunos sectores militares y políticos que consideran que las demarcaciones de tierras indígenas son un riesgo que pueden llevar a la balcanización de la nación. 


\section{La demarcación de tierras indígenas desde los instrumentos legales}

14 En Venezuela, hay que señalar que la promulgación de los instrumentos jurídicos que reconocen los derechos de los pueblos indígenas es sin duda alguna un importante avance en materia indígena. La Constitución Nacional de 1999 (CRBV 1999), la Ley de Demarcación y Garantía del Hábitat y Tierras de los Pueblos Indígenas del 2001, la Ley Orgánica de Pueblos y Comunidades Indígenas del 2005 (LOPCI 2005), la Ley de Idiomas Indígenas (2008), entre otras, hacen referencia directa al reconocimiento de la organización social y política, sus costumbres, idiomas, así como a los derechos indígenas sobre las tierras donde habitan y han habitado. El Artículo 119 de la Constitución Nacional explícitamente reconoce los derechos originarios que tienen los indígenas sobre sus hábitats y tierras «que ancestral y tradicionalmente ocupan y que son necesarias para desarrollar y garantizar sus formas de vida ». Así mismo, Venezuela, en materia internacional ratificó el Convenio 169 sobre Pueblos Indígenas y Tribales de la OIT en 2001, y la Declaración de las Naciones Unidas sobre los derechos de los pueblos indígenas sancionada en 2007. Ambos instrumentos legales exhortan a los Estados a respetar la importancia que tienen para las culturas indígenas su relación con la tierra y sus hábitats.

15 A pesar de que la población total indígena en Venezuela representa un 2,8 por ciento según el censo de población de 2011 (INE 2011), el Estado ha reconocido los derechos de más de 40 pueblos indígenas. Esta apertura hacia la inclusión social desde el punto de vista jurídico, hace que la Constitución de la República Bolivariana de Venezuela sea vista como una de las más avanzadas en cuanto al reconocimiento de los derechos de los pueblos indígenas en América Latina (Mansutti 2000, Bello 2005, Mosonyi 2009).

De igual forma, la Ley Orgánica de Pueblos y Comunidades Indígenas (LOPCI), decretada el 27 de diciembre del 2005, además de reconocer y proteger la existencia de los pueblos indígenas, también garantiza los derechos originarios y colectivos sobre las tierras que los indígenas ancestralmente ocupan para el desarrollo de sus formas de vida. Esta Ley establece las pautas y requerimientos a seguir para la demarcación de hábitats y tierras que contempla la participación de los pueblos y comunidades indígenas en la elaboración de los expedientes que incluyen a su vez los mapas mentales de sus territorios. Todos estos documentos son considerados oficiales al momento de solicitar el reconocimiento de sus tierras. La mencionada Ley da cuenta de al menos tres aspectos relevantes en cuanto a la relación entre el Estado y las poblaciones indígenas en Venezuela: 1) jurídicamente se establece que sin tierras no hay garantía de las formas de vida indígena; 2) la demarcación es un asunto de Estado que se realizará con la participación de los indígenas; y 3) una vez que sean otorgados los títulos colectivos de tierras, estos serán inalienables, inembargables e intransferibles. Este marco jurídico que denota un sentido plural y multiétnico, pareciera que no sólo garantiza sino blinda los derechos originarios sobre las tierras que los indígenas ancestralmente han ocupado (Caballero Arias 2007).

Sin embargo, a pesar de la difusión de estos instrumentos legales y de los esfuerzos de los pueblos indígenas en llevar adelante sus proyectos de " autodemarcación ", el proceso de demarcación territorial ha sido lento, complicado y hasta inoperante para algunos pueblos indígenas (Mansutti 2006, Caballero Arias 2007, Mosonyi 2009). En este contexto de las demandas de los derechos indígenas, nos preguntamos si esas dificultades y demoras en la delimitación de tierras indígenas en Venezuela, tienen que ver solamente 
con la falta de voluntad política del gobierno y la desarticulación o coaptación de los movimientos indígenas venezolanos, o también han sido producto de visiones geopolíticas sesgadas que creen que la idea de "nación» como entidad espacial unificadora está en riesgo de fragmentación. Uno de los argumentos recurrentes que ciertos sectores militares y políticos esgrimen, y que ha llevado a desestimar las demarcaciones indígenas en Venezuela, es el infundado temor al secesionismo del territorio nacional que supuestamente podría amenazar la soberanía nacional del país (Silva Monterrey 2009, Tillet 2012).

En este caso, proponemos que las demarcaciones y titulaciones de tierras indígenas han interpelado la concepción integradora e indivisible de "comunidad imaginada» (Anderson 1993) que tanto el Estado bolivariano como la sociedad civil han construido de la nación venezolana. Siguiendo la premisa de Chatterjee (2008) sobre múltiples visones de nación que se han elaborado en la modernidad, vemos que estas experiencias de delimitación territorial dentro de los marcos jurídicos establecidos por el Estado bolivariano constituyen expresiones heterogéneas en las que se articulan los referentes indígenas autóctonos con visiones más amplias de la nacionalidad venezolana.

Recordemos que la definición clásica de soberanía dentro del ámbito político refiere al poder supremo del Estado y la autoridad que éste ejerce en un territorio. Con base en esta idea general, las demarcaciones tierras indígenas pudieran ser interpretadas como un peligro a la soberanía e integridad del territorio nacional. Sin embargo, estos prejuicios y equívocos en torno a las demarcaciones lucen totalmente contrarios al Artículo 126 de la Constitución Nacional que señala:

«Los pueblos indígenas, como culturas de raíces ancestrales, forman parte de la Nación, del Estado y del pueblo venezolano como único, soberano e indivisible. De conformidad con esta Constitución tienen el deber de salvaguardar la integridad y la soberanía nacional ».

Para ampliar esta discusión sobre el rol de los indígenas en la nación y la integridad nacional, revisaremos algunos conceptos claves y artículos expuestos en la Ley Orgánica de Pueblos y Comunidades Indígenas (LOPCI 2005), los cuales evidencian que la demarcación de tierras indígenas es un derecho colectivo establecido jurídicamente, y que no debe considerarse como expresión de reconocimiento territorial que atente contra la soberanía nacional. Como ha sido señalado por otros autores, el reconocimiento de los derechos indígenas sobre la tierra no cuestiona la soberanía del Estado (Silva Monterrey 2009).

\section{Pueblos y comunidades indígenas, tierras y hábitats indígenas}

21 Hasta finales de 2014, el Estado venezolano ha entregado un total de 93 títulos colectivos de tierra a «comunidades indígenas» de los pueblos kariña, pumé, jivi, cuiba, warao, cumanagoto, mapoyo, hoti y yukpa, entre otros ${ }^{8}$. Estas comunidades indígenas beneficiadas por la titulación de tierras comprenden una población aproximada de 70.000 indígenas, de acuerdo a cifras extraoficiales manejadas por algunas instituciones públicas que trabajan en asuntos indígenas. Hasta ahora ningún título de propiedad colectiva de tierras se ha entregado a un " pueblo indígena » a pesar de las solicitudes realizadas, por ejemplo, por los indígenas pemón y yekuana del estado Bolívar. De las tierras asignadas sólo a "comunidades indígenas", los kariñas del estado Anzoátegui han tenido 
posiblemente las demarcaciones de mayor extensión. Por ejemplo, a la comunidad de Tabero se le asignó 62.939 ha y a Santa Rosa de Tacata le correspondió 40.760 ha según datos aportados por el Ministerio del Poder Popular para el Ambiente. Estas titulaciones de tierra corresponden al 2,39 por ciento del territorio total del estado Anzoátegui. Si tomamos en cuenta que el censo del 2011 estimó que la población indígena en Venezuela era de 724.592 individuos, quiere decir que desde que se promulgó la Ley de Demarcación y Garantía del Hábitat y Tierras de los Pueblos Indígenas en el 2001 cerca de un 9,5\% de los indígenas se han favorecido con la titulación de tierras.

Entendemos que este es un proceso complejo y que requiere no solo de la aplicación adecuada de políticas públicas indígenas en materia de tierras sino también de voluntades políticas y de una mayor coordinación entre las instituciones competentes y los representantes de los pueblos indígenas. Luego de un periodo de casi seis años de estancamiento, entre el 2006 y el 2011, en el que poco se avanzó en cuanto al otorgamiento de títulos de tierras indígenas ${ }^{9}$, las gestiones administrativas y convocatorias para el proceso de la demarcación territorial se reiniciaron en el 2012 en un clima que se encontraba saturado por las campañas y promesas electorales en la que el entonces presidente Hugo Chávez buscaba la reelección presidencial. Así, se reactivaron con cierto impulso las comisiones de trabajo en relación con el proceso de demarcación territorial coordinado por el Ministerio del Poder Popular para los Pueblos Indígenas ${ }^{10}$.

Más allá de estas movilizaciones por parte de las instituciones y los mismos representantes indígenas en el proceso de delimitación de las tierras, vale la pena resaltar que existe un recelo por parte de sectores militares y políticos en cuanto a las demarcaciones de tierras, sobre todo las solicitadas bajo la categoría de "pueblos indígenas », al considerarlas una amenaza a la soberanía nacional por su amplia extensión territorial. Consideramos que esto se debe, entre otros aspectos, a la dificultad en comprender los conceptos claves que presenta la Ley Orgánica de Pueblos y Comunidades Indígenas (LOPCI 2005), en particular sobre la definición de los conglomerados indígenas y su vínculo directo a la dimensión espacial, en este caso referido a las tierras y hábitats.

24 Como hemos señalado, hasta el momento no se han otorgado títulos de tierra a ningún " pueblo indígena » en Venezuela, solo a « comunidades indígenas » de algunas etnias en diferentes estados regionales. Analizaremos cómo la LOPCI define estas dos categorías y cómo se interpretan en relación con la dotación y titulación tierras indígenas. Por «pueblos indígenas » se entiende a los: « ... grupos humanos descendientes de los pueblos originarios que habitan en el espacio geográfico que corresponde al territorio nacional, de conformidad con la Constitución de la Republica Bolivariana de Venezuela y las leyes, que se reconocen a sí mismos como tales, por tener uno o algunos de los siguientes elementos: identidades étnicas, tierras, instituciones sociales, económicas, políticas, culturales y sistemas de justicia propios, que los distinguen de otros sectores de la sociedad nacional y que están determinados a preservar, desarrollar y transmitir a las generaciones futuras » (Artículo 3, numeral 1, LOPCI 2005).

Mientras que las « comunidades indígenas»:

«Son grupos humanos formados por familias indígenas asociadas entre sí, pertenecientes a uno o más pueblos indígenas, que están ubicadas en un determinado espacio geográfico y organizados según las pautas culturales propias de cada pueblo, con o sin modificaciones provenientes de otras culturas » (Artículo 3 , numeral 2, LOPCI 2005). 

grupos humanos, en este caso indígenas, que comparten modos de vida y elementos socio-culturales distintos al resto de la « sociedad nacional». Son sujetos culturalmente diferenciados que al mismo tiempo forman parte del Estado-nación como ciudadanos con deberes y derechos, lo que indica que también son responsables de salvaguardar la integridad de la nación. Sin embargo, más allá de los elementos culturales intangibles o inmateriales a los que hacen alusión las dos definiciones, los pueblos y las comunidades se ubican en espacios geográficos que difieren en cuanto a su dimensión espacial y políticoadministrativa, pero que en ningún momento atentan contra la soberanía nacional, pues en ambos casos hacen alusión de manera referencial al territorio nacional.

En cuanto a las " comunidades indígenas », éstas están formadas por familias indígenas asociadas entre sí que habitan un espacio geográfico determinado, lo cual indica que son grupos humanos de menor población y están circunscritos a espacios de menor extensión territorial. He allí la razón por la cual las demarcaciones que se han concretado oficialmente hasta ahora, han sido sólo a " comunidades indígenas ». Es decir, existe una correlación entre la categoría de comunidades indígenas y su superficie espacial más pequeñas que pueden ser controladas por parte del Estado. Sobre los «pueblos indígenas » resulta ser una categoría mucho más vasta en cuanto a número de población y extensión territorial, con lo cual se percibe como un peligro a la estabilidad de la soberanía nacional, a pesar de que su definición contradice esta percepción.

Por otra parte, el concepto de "pueblos indígenas» reconoce que la «tierra» es un elemento identitario que comparte un grupo humano que tiene ascendencia con los pueblos originarios, es decir poblaciones pretéritas que habitaban antes de los procesos de conquista y colonización europea. Por tanto, este concepto reconoce que hay una relación consubstancial entre la continuidad histórica de los pueblos indígenas actuales con los originarios a través de un recurso como es la tierra. Lo que pretendemos destacar aquí es que aunque la LOPCI reconoce a los "pueblos indígenas » como culturalmente diferentes del resto de la nación, queda claramente expuesto que ellos co-habitan en el territorio nacional de acuerdo a los cánones de la Constitución de la República Bolivariana de Venezuela, es decir dentro de la legalidad y vigilancia de las instituciones del Estado.

30 Advertimos entonces cómo el Estado bolivariano, por el contrario, ha reificado y manipulado la categoría de « comunidades indígenas » con respecto al otorgamiento de títulos de tierra, mientras que desfavorece las demarcaciones por "pueblos", lo cual impide que los indígenas ejerzan su libre determinación como sujetos colectivos y originarios. Esta sobrevalorización de lo comunitario, o «comunitarización de los derechos indígenas» (Angosto 2010: 108), tiene consecuencias múltiples para el desarrollo de las sociedades indígenas. Entre ellas podemos mencionar la división de los espacios étnicos de convivencia, las pugnas y competencias entre líderes, y en general una visión fragmentada de los espacios territoriales y las identidades entre los indígenas.

Para complementar esta discusión sobre las definiciones de los conglomerados humanos que la LOPCI identifica como pueblos y comunidades indígenas, se hace necesario analizarlos en relación con otro par de definiciones que establecen las características de los espacios que ocupan las comunidades y pueblos indígenas. Se trata de los conceptos « tierras indígenas » $\mathrm{y}$ « hábitat indígena ». De acuerdo a esta ley, las « tierras indígenas »:

«Son aquellas en las cuales los pueblos y comunidades indígenas de manera

individual o colectiva ejercen sus derechos originarios y han desarrollado 
tradicional y ancestralmente su vida física, cultural, espiritual, social, económica y política. Comprenden los espacios terrestres, las áreas de cultivo, caza, pesca, recolección, pastoreo, asentamientos, caminos tradicionales, lugares sagrados e históricos y otras áreas que hayan ocupado ancestral o tradicionalmente y que son necesarias para garantizar y desarrollar sus formas específicas de vida » (Artículo 3, numeral 4, LOPCI, 2005).

Por « hábitat indígena » se refiera a:

«... el conjunto de elementos físicos, químicos, biológicos y socioculturales, que constituyen el entorno en el cual los pueblos y comunidades indígenas se desenvuelven y permiten el desarrollo de sus formas tradicionales de vida. Comprende el suelo, el agua, el aire, la flora, la fauna y en general todos aquellos recursos materiales e inmateriales necesarios para garantizar la vida y desarrollo de los pueblos y comunidades indígenas » (Artículo 3, numeral 5, LOPCI, 2005).

Sobre estas dos categorías, lo que hay que resaltar es que mientras el concepto de «tierras indígenas » refiere a una noción de espacio aferrada a la idea de ancestralidad para que los pueblos y comunidades indígenas ejerzan sus derechos en función de su continuidad cultural; el de " hábitat indígena » resalta una serie de elementos y recursos materiales e inmateriales que son necesarios para garantizar los modos de vida de los indígenas. Es decir, el primer concepto alude a la dimensión histórica de los lugares, dominios o áreas donde colectiva $\mathrm{y}$ ancestralmente los indígenas han habitado identificando sus actuales actividades de subsistencia y patrones de asentamiento. El segundo concepto describe la dimensión natural y simbólica de los elementos constitutivos de esos espacios desde una visión casi fenomenológica. En ninguno de los dos casos, el contenido de estas definiciones transgrede las nociones de soberanía nacional o geopolítica nacional. Por el contrario, al resaltar los elementos culturales de las prácticas indígenas en sus tierras, la geografía social se hace más explícita y el conocimiento de los territorios más evidente desde una visión cognitiva y de integración territorial.

De acuerdo a estas definiciones y enunciados es que se rige el proceso de demarcación y titulación de tierras indígenas en Venezuela, lo cual nos lleva a confirmar que no existe contradicción alguna entre el reconocimiento de las tierras indígenas y la gobernabilidad y la soberanía nacional. Esta aseveración se complementa con lo expuesto en el Artículos 10 de esta Ley. Sobre la « integridad territorial » señala que:

«El reconocimiento por parte del Estado de los derechos y garantías contenidas en esta Ley no significa bajo ninguna circunstancia que se autorice o fomente acción alguna encaminada a quebrantar o menoscabar, total o parcialmente, la integridad territorial, la soberanía y la independencia política del Estado venezolano, ni otros principios, derechos y garantías contenidos tanto en la Constitución de la República Bolivariana de Venezuela como en las demás leyes de la República » (Artículo 10, LOPCI, 2005).

Como se puede apreciar, el temor que tienen algunos grupos militares y políticos sobre un fraccionamiento o secesionismo del territorio nacional es injustificado al analizar algunos de los conceptos claves de esta Ley que atañen al reconocimiento de los derechos indígenas sobre sus tierras. Por el contrario, la declaración, dotación y titulación de tierras indígenas no solo garantiza la continuidad cultural de los pueblos indígenas sino que integra y fortalece la soberanía nacional en el sentido de la pluriculturalidad. Seguidamente, veremos cómo los mapas mentales o culturales, que son uno de los requisitos para conformación de los dossiers para las demarcaciones de tierras indígenas, tampoco reflejan criterios contrarios a las visiones geopolíticas de la nación. 


\section{La autodemarcación como expresión cartográfica de la nación}

36 En las expresiones gráficas de representación territorial, las cartografías sociales indígenas se han convertido en una herramienta colectiva que permiten reflejar la dimensión material e inmaterial de los pueblos a través de mapas mentales o culturales. Tal como lo plantea Almeida (2013), las cartografías sociales van mucho más allá de las cartografías oficiales o nacionales. Ellas, constituyen la apropiación de una técnica de representación espacial que permite reivindicar el derecho a las tierras y el acceso a los recursos naturales por parte de grupos sociales en desventaja. Sin embargo, también es cierto que esta visión sobre la cartografía social, es sólo un punto de partida tal como lo refieren Muñoz Gaviria y Rodríguez Cáceres (2010), quienes abogan por una visión dinámica de la cartografía social que se redefina continuamente a partir de las prácticas diferenciadas de los actores sociales con respecto a esta herramienta participativa.

En tal sentido, cuando buscamos dar cuenta de la relación y articulación entre las instituciones del Estado y los pueblos indígenas con respecto a la dimensión territorial, podemos afirmar que se producen diversas formas de territorializacion (Caballero Arias y Cardozo 2006). Nos referimos a que en estos procesos de interacción con las instituciones estatales, los indígenas revelan en sus cartografías (mapas culturales) tanto las representaciones cotidianas y simbólicas que tienen sobre sus tierras, como sus percepciones en cuanto a los efectos de las políticas territoriales y de expansión por parte del Estado y de agentes exógenos sobre sus tierras.

Desde que se decretó la Ley de Demarcación y Garantía del Hábitat y Tierras en enero del 2001 (LDTPI 2001), diferentes pueblos y comunidades indígenas en varias regiones del país iniciaron a motu proprio los procesos de demarcación para solicitar la titulación de sus tierras ante las instancias gubernamentales correspondientes. Estas experiencias locales fueron denominadas por los mismos indígenas como procesos de «autodemarcación de tierras » al asumir la responsabilidad de representar sus espacios territoriales por medio de mapas mentales o culturales como lo establece la LOPCI (2005). Las autodemarcaciones son documentos válidos que forman parte de los recaudos que se requieren para conformar los expedientes (dossiers) de demarcación de tierras, y con ellos solicitar la titulación y reconocimiento de sus tierras ante el Estado venezolano.

Esta actividad de mapeo participativo, por lo general se ha llevado a cabo en reuniones, asambleas y talleres de trabajo asistidos por antropólogos(as), abogados(as) y geógrafos (as) que llevan años trabajando con esos grupos y que han colaborado, bajo diferentes enfoques, en la preparación de esos expedientes. En particular, el apoyo técnico de estos profesionales se ha concretado en contribuir en: la elaboración del informe socioantropológico, la metodología para el diseño de los mapas mentales, y fungir como intermediarios entre las comisiones regionales y las comunidades indígenas. Sin embrago, podríamos afirmar que cada experiencia de demarcación territorial es una experiencia única debido a las particularidades étnicas y visiones que tiene cada pueblo y comunidad indígena, así como a la intencionalidad de los actores (indígenas y no indígenas) con respecto a sus interlocutores (funcionarios públicos). En tal sentido, la autodemarcación, como práctica fundamentalmente indígena, denota algunos elementos sociales, culturales y políticos que hay que destacar. 
40 En primer lugar, las autodemarcaciones en Venezuela, como manifestación de las cartografías indígenas, han sido diversas, complejas y cambiantes en cuanto a las técnicas gráficas utilizadas, los conceptos desarrollados y los referentes naturales, sociales y políticos reseñados en los mapas de acuerdo a las visiones culturales que tiene cada pueblo y comunidad indígena. A través de narraciones orales, historias de vida, mitos y relatos, los indígenas han dado cuenta de sus movimientos migratorios, actividades de subsistencia, características ecológicas, y usos simbólicos de sus territorios, lo que ha resignificado sus conocimientos sobre el territorio donde habitan. Así lo reflejan los trabajos de López-Zent et al. (2004) para el caso de los hoti en Amazonas, González y Zent (2006) para los Yabarana en Amazonas, Caballero y Cardozo (2006) para los Yanomami en Amazonas, Sletto $(2009,2010)$ para los Pemón en el estado Bolívar, Croes y Álvarez (2009) para los Warao, Yekuana, Pemón y Yukpa, entre otros.

41 En segundo lugar, si bien la autodemarcación indígena se traduce en una experiencia etnocartográfica que recoge las visiones fenomenológicas locales en el proceso de la demarcación del hábitat y tierras, lo cierto es que esa supuesta autonomía indígena está determinada por los procedimientos y directrices establecidos en la LOPCI. Las autodemarcaciones que se han realizado son producto de un esquema e intencionalidad gubernamental que se han establecido dentro del proceso de demarcación de tierras guiado por el Estado. Por lo tanto, el proceso de autodemaración no es auto-contenido ni neutral, por el contrario está enmarcado en una plataforma normativa y sistémica del Estado.

42 En este sentido, al analizar los mapas mentales indígenas que se han producido hasta ahora, además de apreciar elementos ecológicos y etnográficos como: el curso de los ríos (por ejemplo el Orinoco, Caura y Caroní), los sitios sagrados, las áreas de caza y de pesca, los tipos de cultivo en los conucos, la ubicación de las aldeas, y variedad ecológica de selvas y sabanas, entre otros aspectos; también se aprecia una visión más políticanacional del territorio. Nos referimos a que en algunos casos, los indígenas también han dibujado en sus mapas elementos idiosincráticos que tienen que ver con la idea del Estado-nación venezolano. Nos referimos, por ejemplo al señalamiento de las parroquias y municipios, la ubicación espacial de las alcaldías, ambulatorios, y escuelas; la identificación de áreas de concentración de poder estatal y zonas de conflicto, e incluso en algunos mapas muestran los trazos de las líneas fronterizas que dividen a Venezuela de Brasil y Colombia. Estos elementos claramente hacen distinción de la dimensión nacional del territorio y revelan la conceptualización que los indígenas van construyendo en torno a la soberanía.

43 Estos ejercicios de cartografiar sus hábitats y tierras, han servido para que los indígenas estén consientes del significado material y simbólico que tienen los espacios que ocupan, pero también para construirse una idea de ciudadanía dentro del Estado-nación. Por consiguiente, la demarcación del hábitat y tierras de los pueblos y comunidades indígenas, nos lleva a considerar una nueva dimensión conceptual que integra las nociones de tierra, indígenas y soberanía nacional. De allí, proponemos que esos espacios delimitados en las cartografías indígenas sean entendidos como espacios construidos social e históricamente, en los cuales los indígenas reflejan una continuidad cultural a través de la producción y reproducción de los patrones culturales. De igual forma, reflejan sus dinámicas y relaciones sociales en base con las formas de territorializacion expresadas en los diferentes grados de integración y articulación con su entorno local, regional y nacional, y reafirman sus derechos como indígenas y ciudadanos venezolanos. 
Vemos entonces como las cartografías indígenas también pueden dar cuenta de la dimensión geopolítica del territorio y reflejar criterios y conocimientos sobre la soberanía y el ordenamiento político-administrativo de la nación venezolana.

\section{Reflexiones finales}

En las últimas dos décadas, los pueblos indígenas de América Latina han transformado su condición de grupos dominados, asimilados y olvidados en fuerzas colectivas, articuladas y visibilizadas en los contextos de los Estados-naciones. Este cambio de estatus dentro de los ámbitos nacionales es el resultado de acciones sociales concretas que se han traducido en la conformación de organizaciones y movimientos indígenas, el surgimiento de un nuevo liderazgo con matices étnicos y las continuas luchas sociales que reclaman el reconocimiento sobre los derechos indígenas en cuanto a tierras, salud, educación y participación en las esferas políticas. Estas formas de resistencia y protestas indígenas fueron decisivas para que muchos de los países latinoamericanos llevaran a cabo importantes reformas en sus constituciones nacionales reconociendo la diversidad cultural y lingüísticas de los pueblos indígenas. Estas constituciones no sólo reconocen la existencia de grupos culturalmente diferentes sino el hecho de que ellos, como pueblos indígenas, tienen derechos colectivos y originarios fundamentados en sus tradiciones históricas y ancestrales. Sus lenguas, costumbres, sistemas políticos, cosmologías, organizaciones sociales y territorios son algunos aspectos que estos marcos legales han reconocido acordes con las particularidades de la diversidad indígena en cada nación.

No hay duda que los movimientos indígenas en los inicios del siglo 21 se han convertido en importantes fuerzas étnicas y políticas que reclaman sus derechos como pueblos y se esfuerzan por alcanzar una mayor participación en las esferas públicas y políticas por medio de diversas estratégicas heurísticas de disputa. A pesar de estos importantes cambios que se reflejan en las reformas constitucionales, en la promulgación de nuevos instrumentos jurídicos que avalan los derechos indígenas en áreas especificas, en la proclamación de la Declaración de las Naciones Unidas sobre los Derechos de los Pueblos Indígenas (ONU 2007), y en el hecho de que representantes indígenas ocupan posiciones cada vez más relevantes en la política; los pueblos indígenas a través de sus organizaciones aún demandan y luchan por el ejercicio de los derechos sobre la tierra y la libre determinación.

La tierra, la Pachamana, se ha convertido en el objetivo central de las movilizaciones indígenas en los últimos años, pues no ha sido suficiente con el reconocimiento jurídico de los derechos indígenas en los dominios sociales e intangibles. La concreción y el valor político-económico que tiene la tierra en las naciones de América Latina, la ha convertido en un bien, en un « super don » de gran demanda. No obstante; esas tierras han sido y son territorios indígenas. La pluriculturalidad y las nuevas formas de inclusión social llegaran a ser evidentes e incontestables cuando los derechos de los pueblos indígenas sobre sus tierras no queden como simples apéndices en un universo de normas y legalidades.

En estos apuntes sobre los procesos de reconocimiento, demarcación y titulación de tierras indígenas en Venezuela, hemos visto que no basta con el desarrollo de sendos marcos jurídicos que abalen el reconocimiento y la diversidad cultural para que se implementen políticas públicas indígenas en cuanto a sus tierras. A demás de procurar el mejoramiento de las bases institucionales cónsonas con el reconocimiento de los derechos de los pueblos indígenas, se deben simplificar los criterios técnicos de la demarcación 
territorial y reactivar de manera responsable las comisiones de trabajo, tal como hemos señalado en trabajos anteriores (Caballero Arias y López-Zent 2006, Caballero Arias 2007). Asimismo, hace falta propiciar una real y directa articulación y diálogo entre las instituciones del Estado y las poblaciones indígenas en cuanto a la delimitación de las tierras de acuerdo a sus especificidades socio-culturales y a sus nociones de territorialidad que son representadas en sus cartografías o mapas culturales. El establecer puentes y conexiones entre sistemas culturales diferentes, es decir entre la sociedad nacional venezolana y los pueblos indígenas, ha resultado ser todo un reto para las instituciones del Estado, en tanto que les resulta difícil adoptar y aplicar los enfoques del pluralismo jurídico al que hace alusión la Constitución Nacional, sobre todo en cuanto al reconocimiento de las tierras indígenas.

El caso de Venezuela que hemos aquí discutido, ha mostrado que se debe procurar crear espacios más interculturales para el diálogo y la articulación de propuestas institucionales e indígenas en concordancia con las particularidades socio-culturales de cada pueblo indígena y con sus múltiples nociones de territorialidad. El derecho a la demarcación territorial indígena en Venezuela debe trascender la esfera jurídica y entenderse como una manifestación inédita que permita dinamizar las perspectivas en torno al ordenamiento territorial desde una visión de la interculturalidad. En vez de verla como una amenaza a la soberanía nacional, hay que entenderla como parte de las nuevas formas de organización geopolítica que los Estados-naciones multiétnicos y pluriculturales procuran construir en los albores del siglo 21 a partir de una visión heterogénea de la nación.

\section{BIBLIOGRAFÍA}

Almeida A.W. 2013 - Pueblos y comunidades tradicionales. Nueva cartografía social. Manaos, UEA Ediciones, $176 \mathrm{p}$.

Anderson B. 1993 [1983] - Comunidades imaginadas. Reflexiones sobre el origen y la difusión del nacionalismo. México, DF., Fondo de Cultura Económica.

Angosto L.F. 2010 - Pueblos indígenas, multiculturalismo y la nueva geometría del poder en Venezuela. Cuadernos Cendes 27 (73) : 98-132.

Arvelo-Jiménez N. \& Jiménez S. (Ed.) 2001 - Atlas Dekuana. Caracas, Asociación Otro Futuro.

AVN (Agencia Venezolana de Noticias) 2011 - Recuperadas más de 15.800 hectáreas para comunidades indígenas yukpa. En: AVN 12/10/2011. [Documento electrónico : http:// www.avn.info.ve/contenido/recuperadas-m\%C3\%A1s-15800-hect\%C3\%A1reas-paracomunidades-ind\%C3\%ADgenas-yukpa, revisado 30 de junio, 2015].

Bajtin M. 1989 - Teoría y estética de la novela. Madrid, Taurus, 511 p.

Bello L. 2005 - Derechos de los Pueblos Indígenas en el Nuevo Ordenamiento Jurídico. Copenhague, IWGIA, 487 p. (IWGIA doc ; 52). 
Caballero Arias H. 2007 - La Demarcación de Tierras Indígenas en Venezuela. Revista de Ciencias Económicas y Sociales 13 (3) : 189-208.

Caballero Arias H. \& Cardozo J.I. 2006 - Políticas territoriales, identidad y memoria histórica: Los Yanomami ante la demarcación de sus tierras. Antropológica 105-106 : 99-130.

Caballero Arias H. \& López-Zent E. 2006 - Introducción. Antropológica 105-106 : 5-12.

Chatterjje P. 2008 - La nación en tiempo heterogéneo y otros estudios subalternos. Buenos Aires, Siglo XXI, 296 p.

Croes G. \& Álvarez T. 2009 - Cartografías indígenas. Caracas, PNUD-Venezuela y Anthropos, 47 p.

CRBV (Constitución Nacional de la República Bolivariana de Venezuela) 1999 - Constitución Nacional de la República Bolivariana de Venezuela. Caracas : Imprenta Nacional.

GBM (Grupo Banco Mundial) 2015 - Latinoamérica Indígena en el Siglo XXI. Washington DC, Banco Internacional de Reconstrucción y Fomento/Banco Mundial, 120 p.

González J. \& Zent S. 2006 - Experiencias en el proceso de demarcación de hábitat y tierras de las comunidades multi-étnicas Yabarana-Jotï-Panare-Piaroa del sector Parucito-Manapiare-Yutaje, Edo. Amazonas, Venezuela. Antropológica 105-106 : 41-65.

INE (Instituto Nacional de Estadísticas) 2011 - Resultados población indígena. XVI Censo de Población y Vivienda 2011. [Documento electrónico : http://www.ine.gob.ve/documentos/Demografia/ CensodePoblacionyVivienda/pdf/ResultadosBasicos.pdf].

LDTPI (Ley de Demarcación) 2001 - Ley de Demarcación y Garantía del Hábitat y Tierras de los Pueblos Indígenas. Gaceta Oficial 37.118 del 12 de Enero del 2001. República Bolivariana de Venezuela.

Ley de Idioma Indígenas 2008 - Ley de Idiomas Indígenas. Gaceta Oficial No 38981, 28 de julio, 2008. Caracas, Venezuela.

LOPCI (Ley Orgánica de Pueblos y Comunidades Indígenas) 2005 - Ley Orgánica de Pueblos y Comunidades Indígenas. Gaceta Oficial 38.344 del 27 de diciembre del 2005. República Bolivariana de Venezuela.

López Zent E., Zent S. \& Marius L. 2004 - Autodemarcando la Tierra: Explorando las Ideas, los Árboles y Caminos Hotï. Boletín Antropológico 59 (3) : 313-338.

Mansutti A. 2000 - Pueblos indígenas y cambios constitucionales: el caso de Venezuela. Boletín Antropológico 50 : 79-98.

Mansutti A. 2006 - La demarcación de territorios indígenas en Venezuela: algunas condiciones de funcionamiento y el rol de los antropólogos. Antropológica 105-106 : 13-38.

Ministerio del Poder Popular de los Pueblos Indígenas (Minpi) 2013-2014 - Memorias y Cuenta 2013-2014. [Documento electrónico : http://www.minpi.gob.ve/index.php/menpgestion/ menpgmemoria, revisado el 1 de abril, 2016].

Monsonyi E. 2009 - Balance general de los diez años del proceso bolivariano. Avances y contradicciones. Revista Venezolana de Economía y Ciencias Sociales 15 (1) : 155-172.

Muñoz Gavíria E. \& Rodríguez Cáceres L. 2010 - Cartografía social y territorio en América latina Memorias del seminario de Rio de janeiro, julio 2010. Antropológica 114 : 129-159.

OIEA (Organizaciones Indígenas del Estado Amazonas) 2011 - Pronunciamiento Organizaciones Indígenas Amazonas sobre nuevo decreto Comisión Demarcación. En: Forest Peoples Programme, 22 de marzo, 2011. [Documento electronic : http://www.forestpeoples.org/topics/rights-land- 
natural-resources/news/2011/03/venezuela indigenous-peoples-speak-out-against-yet, revisado 25 de junio, 2015].

ONU (Organización de las Naciones Unidas) 2007 - Declaración de las Naciones Unidas sobre los Derechos de los Pueblos Indígenas. [Documento electrónico : http://www.un.org/esa/socdev/unpfii/ documents/DRIPS_es.pdf, revisado el 15 de marzo, 2016].

Portillo L. 2011 - Palabras de Lusbi Portillo en la Comisión Interamericana de Derechos Humanos de la Organización de Estados Americanos, Washington, DC. En: Clorofilazul. [Documento electrónico : http://clorofilazul.blogspot.com/2011/03/palabras-de-lusbi-portillo-en-la.html, revisado el 15 de julio, 2015].

Romero Z. 2012 - Documento presentado por la dirigente del pueblo yukpa, Zenaida Romero, Hija de Sabino Romero en la ONU. En: Homo et Natura, 06 de mayo, 2012. [Documento electrónico : http://www.aporrea.org/ddhh/a142986.html, revisado el 12 de julio, 2015].

Silva Monterrey N. 2009 - Reconocimientos Territoriales Indígenas y Soberanía del Estado en Venezuela. Kuawäi. Revista Arbitrada del Departamento Hombre y Ambiente 2 (3) : 32-46.

Sletto B. 2009 - « We drew what we imagined » : participatory mapping, performance and the arts of landscape-making. Current Anthropology 50 : 443-476.

Sletto B. 2010 - Autogestión en representaciones espaciales indígenas y el rol de la capacitación y concientización: el caso del Proyecto Etnocartográfico Inna Kowantok, Sector 5 Pemón (Kavanayén-Mapauri), La Gran Sabana. Antropológica 113 : 43-75.

Tillet A. 2012 - Venezuela. En: Mikkelsen C. (Ed.) El Mundo Indígena. Copenhague, Grupo Internacional de Trabajo sobre Asuntos Indígenas IWGIA : 126-13.

UIV (Universidad Indígena de Venezuela) 2011 - ¡Seguimos vivos! ¡Somos colectivos! ¡Resistimos! Comunicado de la Universidad Indígena de Venezuela a los venezolanos y al mundo en la Semana de la Resistencia. En: Nodanakaroda, 12 de octubre, 2011. [Documento electrónico : http:// rbaniwa.wordpress.com/2011/10/27/\%C2\%A1seguimos-vivos-\%C2\%A1somos-colectivos-\%C2\% A1resistimos/, revisado 25 de junio, 2015].

\section{NOTAS}

1. Sobre experiencias de cartografías indígenas en Venezuela, ver por ejemplo los trabajos de: Arvelo-Jiménez y Jiménez (2001), López-Zent et al. (2004), González y Zent (2006), Sletto (2009), entre otros.

2. El informe Latinoamérica Indígena en el Siglo XXI (GBM 2015) advierte sobre estas nuevas formas de expansión neocolonial y reporta, por ejemplo, que la quinta parte de la cuenca Amazónica tiene potencial minero, de los cuales un $20 \%$ de la región son tierras indígenas que están siendo amenazadas por la extracción ilegal de oro.

3. La sustitución del nombre "Día de la Raza » por el «Día de la Resistencia Indígena " fue establecida oficialmente el 11 de octubre de 2002, según decreto No. 2028 sancionado por la Asamblea Nacional de Venezuela.

4. El otorgamiento de títulos de tierras se ha hecho solamente a «comunidades indígenas ", en ninguno de los casos el Estado ha entregado títulos de tierra a "pueblos indígenas " como conglomerados étnicos. De acuerdo a los últimos datos de las Memorias y Cuentas del Ministerio del Poder Popular para los Pueblos Indígenas, entre el 2013 y 2014, se entregaron sólo 27 títulos a comunidades indígenas, los cuales representan una extensión de 1.134.349 ha. (Minpi 2013, 2014). 
5. Ante las presiones de los movimientos indígenas durante el 2011, el entonces vicepresidente de la república Elías Jahua anunció que 15.800 hectáreas, de 25 haciendas, estarían bajo el resguardo del Gobierno Nacional para iniciar el proceso de delimitaciones de las tierras yukpa en la Sierra de Perijá, en el estado Zulia (AVN 2011).

6. Tal es el caso del luchador y líder indígena yukpa Sabino Romero, de la comunidad de Chaktapa, Sierra de Perijá, estado Zulia, quien fuera asesinado el 3 de marzo, 2013.

7. Un ejemplo de ello, es la iniciativa de la ONG « Territorio Indígena y Gobernanza » en Bolivia (ver http://www.territorioindigenaygobernanza.com).

8. Datos obtenidos de las « Memorias y Cuentas» del Ministerio del Poder Popular de los Pueblos Indígenas, http://www.minpi.gob.ve/index.php/menpgestion/menpgmemoria y de Caballero Arias (2007).

9. Entre 2006 y 2011, solo se entregaron 22 títulos de tierras siguiendo el criterio de « comunidades indígenas » de acuerdo a lo establecido en la LOPCI (2005).

10. Hasta finales del 2010, la coordinación de la Comisión Nacional de Demarcación del Hábitat y Tierra de Pueblos y Comunidades Indígenas estuvo a cargo del Ministerio para el Poder Popular para el Ambiente. Luego, de acuerdo al decreto presidencial No 7.855 del 26 de noviembre, 2010, que apareció en la Gaceta Oficial No 39.624 se aprobó la «Reestructuración y Reorganización de la Comisión Nacional de Demarcación de Hábitat y Tierras Indígenas ». Este decreto anunciaba un cambio en la competencia en materia de demarcación de tierras indígenas, la cual pasaría estar a cargo del Ministerio del Poder Popular para los Pueblos Indígenas. Esta modificación y reestructuración de la normativa fue cuestionada por varias organizaciones indígenas en todo el país, al considerarla como una decisión inconsulta que violentaba los derechos indígenas a la participación y a la toma de decisiones en cuanto a la demarcación de las tierras indígenas. Para más datos sobre estos pronunciamientos véase un documento publicado por las Organizaciones Indígenas del Estado Amazonas (OIEA 2011).

\section{RESÚMENES}

Este trabajo plantea explorar la dimensión política de las demandas territoriales indígenas y discutir los procesos de demarcación de tierras y las cartografías indígenas en relación con los marcos jurídicos nacionales y la noción de soberanía nacional en Venezuela. En primer lugar, presentaremos un contexto general de las luchas y estrategias heurísticas de disputa territorial por parte de los indígenas en América Latina en defensa de la Pachamama. Luego discutiremos los marcos jurídicos vigentes y cómo los conceptos que expone la Ley Orgánica de Pueblos y Comunidades Indígenas (LOPCI 2005) en Venezuela en torno a pueblos y comunidades indígenas, por una parte, y tierras y hábitats indígenas por la otra no vulneran las nociones de soberanía nacional, como se ha pretendido establecer. Asimismo, planteamos que los mapas mentales indígenas como requerimientos para hacer la solicitud formal de las tierras ante las instancias gubernamentales reflejan una visión espacial intercultural que toma en cuenta tanto las visiones territoriales indígenas como el ordenamiento espacial y la dimensión geopolítica de la nación venezolana. Los mapas mentales indígenas son objetos de producción socio-histórica que reflejan tanto el sentido patrimonial y estético de los conocimientos locales sobre el espacio y el territorio, como el sentido más político de la idea de nación. 
Cet article explore la dimension politique des revendications territoriales autochtones et analyse les processus de démarcation des terres et de cartographie indigènes en relation avec le cadre des lois et la notion de souveraineté nationale au Venezuela. En premier lieu, nous présentons le contexte général des luttes et des stratégies territoriales des peuples amérindiens en défense de Pachamama en Amérique Latine. Dans les cadres juridiques actuels et la Loi Organique des Peuples et Communautés Indigènes du Venezuela (LOPCI 2005), nous discutons ensuite les concepts de "peuples " et "communautés " indigènes d'une part, et ceux de «terres " et « habitats » indigènes d'autre part, pour montrer qu'ils ne mettent pas en question les notions de souveraineté nationale. Au contraire, il apparait que les cartographies autochtones - exigées ici pour toute demande formelle de démarcation de terre auprès des instances gouvernementales reflètent une vision interculturelle de l'espace capable d'intégrer à la fois les visions territoriales indigènes et la dimension géopolitique de la nation vénézuélienne. Les cartes participatives autochtones sont des objets de production socio-historique qui reflètent à la fois les aspects patrimonial et esthétique des savoirs locaux sur l'espace et le territoire, et le sens le plus politique de l'idée de nation.

This paper explores the political dimension of indigenous territorial demands and the process of land demarcation and indigenous cartographies in relation to national legal frameworks and the notion of national sovereignty in Venezuela. First, we will present a general context of territorial struggle strategies carried out by indigenous people in defense of Pachamama in Latin America. Then, we will disclose the legal frameworks and how the concepts on indigenous peoples, indigenous communities, indigenous lands and indigenous habitats, acknowledged in the Organic Law of Indigenous Peoples and Communities (LOPCI 2005) in Venezuela, do not violate the notion of national sovereignty. Furthermore, we will suggest that indigenous mappings, as requirements to make the formal request for land demarcation to the state, reflect an intercultural spatial vision that takes into account both indigenous territorial perceptions and the geopolitical dimension of the Venezuelan nation. Indigenous participatory mappings are objects of sociohistorical production that reflect both the heritage and aesthetic sense of local knowledge about space and territory as a more political sense of the idea of nation.

\section{ÍNDICE}

Mots-clés: cartographie autochtone, démarcation de terres, cadres légaux, peuples et communautés indigènes, souveraineté nationale

Palabras claves: Cartografías indígenas, demarcación de tierras, marcos legales, pueblos y comunidades indígenas, soberanía nacional, Venezuela

Índice geográfico: Venezuela

Keywords: indigenous cartographies, land demarcation, legal frames, indigenous peoples and communities, national sovereignty, Venezuela

\section{AUTOR}

\section{HORTENSIA CABALLERO ARIAS}

Investigadora Asociada del Laboratorio de Antropología del Desarrollo, Centro de Antropología, Instituto Venezolano de Investigaciones Científicas, IVIC. Email: hcaballe@gmail.com 